\title{
Adaptive Steering Control for Autonomous Lane Change Maneuver*
}

\author{
Plamen Petrov and Fawzi Nashashibi, Member, IEEE
}

\begin{abstract}
In this paper, we present a two-layer nonlinear adaptive steering controller for autonomous lane change maneuver with respect to a stopped vehicle. First, we derive a dynamic model of the vehicle using the Boltzmann-Hamel method in quasi-coordinates for nonholonomic systems. The lane change maneuver is investigated as a tracking problem with respect to desired cycloidal trajectory, which is generated in real time. An adaptive update control law is designed that allows tracking the desired trajectories in the presence of unknown inertial parameters of the vehicle. Simulation results illustrate the performance of the proposed controller.
\end{abstract}

\section{INTRODUCTION}

In recent years, significant advance has been made in designing automated vehicles. The ultimate goal in automating the driving process is to reduce accidents caused by human errors and improve safety. In the same time, the full automation can greatly increase the roadway capacity and diminish air pollution by efficient use of fuel. Fully automated vehicle operation has been investigated, prototyped and demonstrated in several projects during the last decades like Cybercars and CyberCars 2, STARDUST, CyberMove, CityMobil, HAVEit, which address the issue of automated driving like adaptive cruise control, lane keeping, lane change, stop\&go and platooning. The lane change maneuver has been a challenging problem for road automation during the last two decades. The objective of the lane changing is to transfer a vehicle in an adjacent lane which could be also interpreted as an obstacle avoidance maneuver with respect to a moving or a stopped vehicle. For this purpose, vehicle longitudinal and lateral control is required. The automatic control of the longitudinal and lateral motion of the vehicle during the lane changing is often separately undertaken, and each controller is designed as if the longitudinal and steering vehicle dynamics is decoupled. For low speed motion (for speeds less than $5 \mathrm{~m} / \mathrm{s}$ ), which is often the case of automated vehicles, a reasonable assumption is that the slip angles of the wheels are zero, [1], and classical methods from analytic mechanics can be used to develop mathematical models of the vehicle as a nonholonomic system [2,3]. During the lane change maneuver, the vehicle should have predefined (desired) trajectory to track. The existing desired trajectories can be categorized, based on the types of curves that generate: circular [4], harmonic [5], polynomial [6,7] line segments,

* The authors acknowledge the financial support from the ABV project. Plamen Petrov is with the Faculty of Mechanical Engineering, Technical University of Sofia, 1797 Sofia, Bulgaria (e-mail: ppetrov@tu-sofia.bg).

Fawzi Nashashibi is with IMARA, the French National Institute for Research in Computer Science and Control (INRIA) Paris-Rocquencourt, 78153 Le Chesnay, France, (e-mail: Fawzi.Nashashibi@inria.fr). which, in general, should be functions of time. Control techniques as sliding mode [8] and robust switching control [9] have been used for designing lane change controllers.

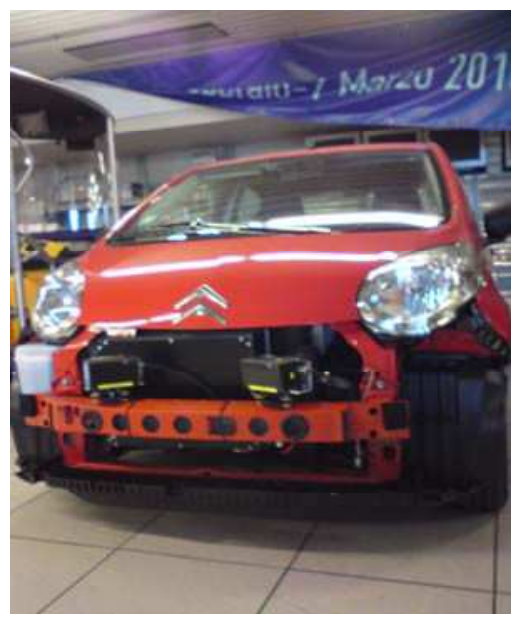

Figure 1. Automated vehicle developed at INRIA equipped with two laser rangefinders

In this paper, we propose a two-layer nonlinear adaptive steering controller for an autonomous lane change maneuver with respect to a stopped vehicle. We consider the problem of autonomous lane change without the use of road infrastructure with only the current inter-vehicle position and orientation available for feedback control which is obtained from onboard sensors. In Fig. 1, is shown an experimental automated vehicle developed at INRIA which is equipped with two laser rangefinders for automatic obstacle avoidance. The organization of the paper is as follows: A dynamic model of the vehicle is derived in Section II. The Problem formulation is given in Section III. In Section IV, a nonlinear adaptive control law is designed. Section V contains simulation results. Conclusions are presented in Section VI.

\section{VEHICLE MODEL}

\section{A. Vehicle Kinematic Model}

In this paper, we consider a front-wheel steering vehicle as shown in Fig. 2. The kinematic scheme of the vehicle represents a two-mass system: the first one consists of a vehicle body comprising the driver and passengers, and the second consists of the two steering wheels including the steering mechanism. It is assumed that the vehicle is moving along flat road at low speed (less than $5 \mathrm{~m} / \mathrm{s}$ ). It is also assumed that the wheels roll without lateral sliding, and the velocity vectors of the wheels are in the direction of the orientation of the wheels, i.e., the wheels are non-deformable. In order to develop a mathematical model of the vehicle 
suitable for control design applications, we consider the so called "bicycle model", where the front steering wheels and the rear wheels are replaced by single virtual wheels at the mid-point $S$ and $P$ of the front and rear vehicle axles, respectively, (Fig. 2). An inertial frame Fxyz is defined in the plane of motion, where the $z$-axis is perpendicular to the road. A body-fixed coordinate frame $P x_{P} y_{P} z_{P}$ is attached at the mid-point $P$ between the rear wheels such that the $x_{P}$ axis is parallel to the vehicle longitudinal base and $y_{P}$ is in the direction of rear wheel axle.

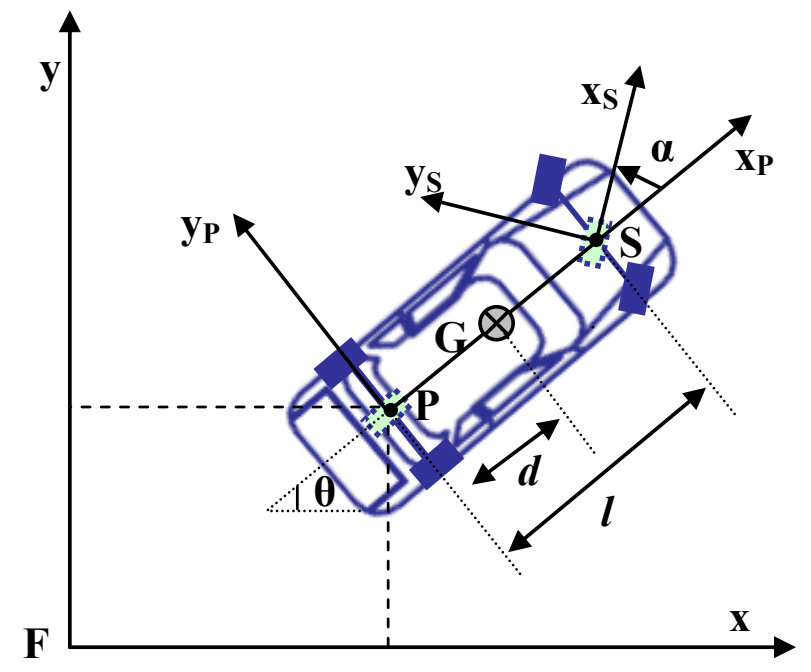

Figure 2. Schematic plan view of a front-wheel steering vehicle

A coordinate system $S x_{S} y_{S}$ is attached to the front virtual wheel and has its origin placed at the center of the wheel, as shown in Fig. 2. The coordinates of the reference point $P$ with respect to an inertial frame Fxyz in the plane are denoted by $\left(x_{P}, y_{P}\right)$. The angle $\theta$ defined as angle between the $x$ and $x_{P}$ axes of $F x y$ and $P x_{P} y_{P}$, respectively, is the orientation angle of the vehicle. The distance between the reference point $P$ and the center of mass of the vehicle body $G$ is denoted by $d$, the vehicle base (the distance between the front and rear vehicle axels) is denoted by $l$, and $\alpha$ is the front-wheel steering angle. If the inertia of the wheels with respect to their proper axes is ignored, using the coordinates of the reference point $P$, the configuration of the vehicle can be described by four generalized coordinates

$$
q=\left[x_{P}, y_{P}, \theta, \alpha\right]^{T} \in R^{4 x 1} .
$$

The system is characterized by the following nonholonomic constraints on the generalized velocities $\dot{q}$

$$
A(q) \dot{q}=0
$$

where the matrix $A \in R^{2 \times 4}$ has the form

$$
A(q)=\left[\begin{array}{cccc}
-\sin (\theta+\alpha) & \cos (\theta+\alpha) & l \cos \alpha & 0 \\
-\sin \theta & \cos \theta & 0 & 0
\end{array}\right],
$$

and the vector $\dot{q} \in R^{4 \times 1}$ is a vector of the generalized velocities. The constraint equations (2) can be converted into an affine driftless control system of the form

$$
\dot{q}=C \eta_{b}
$$

where the columns of matrix $C \in R^{4 \times 2}$

$$
C=\left[\begin{array}{cc}
\cos \theta & 0 \\
\sin \theta & 0 \\
\frac{\tan \alpha}{l} & 0 \\
0 & 1
\end{array}\right]
$$

form a basis of the null space of the matrix $A$. The components of the vector $\eta_{b}$

$$
\eta_{b}=\left[\begin{array}{c}
v_{P x} \\
\omega_{\alpha}
\end{array}\right]
$$

are the so called quasi-velocities and includes the velocity component $v_{P x}$ of point $P$ taken along the axis $P x_{P}$ and the angular velocity $\omega_{\alpha}$ of the front virtual wheel about a vertical axis passing trough point $S$. The quasi-velocities $v_{P x}$ and $\omega_{\alpha}$ can be expressed in terms of the generalize velocities as follows

$$
\eta_{b}=B(q) \dot{q}
$$

where the matrix $B \in R^{2 \times 4}$ has the form

$$
B(q)=\left[\begin{array}{cccc}
\cos \theta & \sin \theta & 0 & 0 \\
0 & 0 & 0 & 1
\end{array}\right] \text {. }
$$

\section{B. Boltzman-Hamel Equations}

In order to derive the dynamic model of the vehicle, we use the Boltzmann-Hamel method [10, 11], which is suitable for systems with nonholonomic constraints. Consider a system, which is subject to $m$ nonholonomic constraints of the form

$$
A(q) \dot{q}=0
$$

where $A \in R^{m x n}$ is the matrix of the constraints, $q \in R^{n x^{1}}$ is a vector of the generalized coordinates of the system, and $\dot{q} \in$ $R^{n \times 1}$ is the vector of the generalized velocities. Let us introduce $n$ expressions for the quasi-velocities $\eta=\left[\eta_{a} \mid\right.$ $\left.\eta_{b}\right]^{\mathrm{T}} \in R^{n \times 1}$ in the following manner

$$
\eta_{a}:=A(q) \dot{q}=0 ; \quad \eta_{b}:=B(q) \dot{q}
$$

where $\eta_{a} \in R^{m \times 1}$ is a vector of the zero quasi-velocities defined by the nonholonomic constraints (9) and $\eta_{b} \in R^{(n-m) \times I}$ is a vector of the chosen non-zero quasi-velocities. Equations (10) can be written in compact form as

$$
\alpha(q) \dot{q}=\eta
$$

where the matrix $\alpha(q) \in R^{n x n}$ has the form

$$
\begin{aligned}
& \alpha(q):=\left[a_{i j}(q)\right]=[A(q)B(q)]^{T} \\
& i=1, \ldots, n ; j=1, \ldots, n
\end{aligned}
$$

The only restriction on the choice of the nonzero quasivelocities $\eta_{b}$ is that the matrix $\alpha(q)$ must be nonsingular.

The general velocities $\dot{q}$ can be expressed in terms of quasi-velocities $\eta$ as follows

$$
\dot{q}=\beta(q) \eta
$$

where the nonsingular matrix $\beta(q) \in R^{n \times n}$ is obtained as follows

$$
\beta(q):=\left[b_{i j}(q)\right]=\alpha^{-1}(q)
$$




$$
i=1, \ldots, n ; j=1, \ldots, n
$$

The Boltzmann-Hamel formulation of the system dynamic equations is given by

$$
\begin{gathered}
\frac{d}{d t} \frac{\partial T}{\partial \eta_{s}}-\frac{\partial T}{\partial \pi_{s}}+\sum_{r=1}^{n} \sum_{t=m+1}^{n} \gamma_{t s}^{r} \frac{\partial T}{\partial \eta_{r}} \eta_{t}=\Gamma_{s} \\
s=m+1, \ldots . ., n
\end{gathered}
$$

where $\pi_{S}$ are the quasi-coordinates, $\left(d \pi_{S} / d t=\eta_{S}\right) ; T(\eta)$ is the kinetic energy of the system expressed in terms of quasivelocities $\eta ; \gamma_{t s}^{r}$ are the three-index coefficients; $\Gamma_{S}$ are generalized forces associated with the quasi-coordinates.

We note that the number of equations in (15) is equal of the number $(n-m)$ of DOFs of the system. Using the elements of the matrices $\alpha(q)$ and $\beta(q)$, the three-index coefficients $\gamma_{t s}^{r}$ and the expressions of the derivatives of the kinetic energy with respect to the quasi-coordinates $\partial T / \partial \pi_{S}$ are determined by using the following relationships

$$
\begin{gathered}
\gamma_{t s}^{r}=\sum_{k=1}^{n} \sum_{l=1}^{n} a_{r l}\left(\frac{\partial b_{l t}}{\partial q_{k}} b_{k s}-\frac{\partial b_{l s}}{\partial q_{k}} b_{k t}\right) \\
\frac{\partial T}{\partial \pi_{s}}=\sum_{r=1}^{n} b_{r s} \frac{\partial T}{\partial q_{r}} .
\end{gathered}
$$

for $t=m+1, \ldots \ldots, n ; r=1, \ldots \ldots, n ; \quad s=m+1, \ldots . ., n$.

\section{Vehicle Dynamic Equations of Motion}

In order to derive the dynamic model of the vehicle, we introduce the following quasi-velocities $\eta \in R^{4}$

$$
\eta=\left[\begin{array}{l:l:l}
\eta_{a} & \eta_{b}
\end{array}\right]^{T}=\left[\begin{array}{ll:l}
\eta_{1}, \eta_{2} & \eta_{3}, \eta_{4}
\end{array}\right]^{T}=\left[\begin{array}{lll}
v_{S y}, v_{P y} & v_{P x}, \omega_{\alpha}
\end{array}\right]^{T}
$$

where $\left(v_{S y}, v_{P y}\right)$ are the velocity components of points $S$ and $P$ taken along the axes $S y_{s}$ and $P y_{P}$, respectively, and $\eta_{3}$ and $\eta_{4}$ are described by (6). In conformity with the nonholonomic constraint (2), $\eta_{1}=\eta_{2}=0$. In this case, the matrices $\alpha$ and $\beta$ introduced in (12), and (14), respectively, look like

$$
\alpha(q)=\left[\begin{array}{cccc}
-\sin (\theta+\alpha) & \cos (\theta+\alpha) & l \cos \alpha & 0 \\
-\sin \theta & \cos \theta & 0 & 0 \\
\cos \theta & \sin \theta & 0 & 0 \\
0 & 0 & 0 & 1
\end{array}\right]
$$

In order to calculate the kinetic energy of the two-mass vehicle in terms of the quasi-velocities (18), we first, express the velocities $\mathbf{v}_{\mathbf{P}}$ and $\mathbf{v}_{\mathbf{S}}$ of points $P$ and $S$, respectively, the angular velocities of the vehicle $\boldsymbol{\omega}$ and the angular velocity of the front virtual wheel $\omega_{\alpha}$ about a vertical axis in terms of quasi-velocities $\eta$ as follows

$$
\begin{aligned}
& \mathbf{v}_{\mathbf{P}}=\left[\begin{array}{lll}
\eta_{3}, & \eta_{2}, & 0
\end{array}\right]^{T} \\
& \boldsymbol{\omega}=\left[\begin{array}{lll}
\frac{\eta_{1}}{l \cos \alpha}, & -\frac{\eta_{2}}{l}, & \frac{\eta_{3} \tan \alpha}{l}
\end{array}\right]^{T} \\
& \boldsymbol{\omega}_{\boldsymbol{\alpha}}=\left[\begin{array}{lll}
0, & 0, & \eta_{4}
\end{array}\right]^{T}
\end{aligned}
$$

$$
\mathbf{v}_{\mathbf{S}}=\mathbf{v}_{\mathbf{P}}+\boldsymbol{\omega} \times \mathbf{l}=\left[\begin{array}{lll}
\eta_{1} \tan \alpha+\frac{\eta_{3}}{\cos \alpha}, & \eta_{1}, & 0
\end{array}\right]^{T}
$$

where $\mathbf{l}=\mathbf{P S},(|\mathbf{l}|=l)$, is the distance vector between the rear and front vehicle axles. Using (21), (22), (23) and (24), the overall kinetic energy of the vehicle is obtained in the form

$$
\begin{aligned}
T= & \frac{1}{2} \mathbf{v}_{\mathbf{P}}^{\mathbf{T}} m_{v} \mathbf{v}_{\mathbf{P}}+m_{v}\left(\mathbf{v}_{\mathbf{P}} \mathbf{x} \boldsymbol{\omega}\right) \mathbf{d}+\frac{1}{2} \boldsymbol{\omega}^{\mathbf{T}} I_{v} \boldsymbol{\omega}+\frac{1}{2} \mathbf{v}_{\mathbf{s}}^{\mathbf{T}} m_{s} \mathbf{v}_{\mathbf{s}} \\
& +\frac{1}{2}\left(\boldsymbol{\omega}+\boldsymbol{\omega}_{\mathbf{\alpha}}\right)^{T} I_{S}\left(\boldsymbol{\omega}+\boldsymbol{\omega}_{\mathbf{\alpha}}\right) \\
= & \frac{1}{2} m_{v}\left(\eta_{2}^{2}+\eta_{3}^{2}\right)+m_{v} d\left(\frac{1}{l}\left(\frac{\eta_{1}}{\cos \alpha}-\eta_{2}+\eta_{3} \tan \alpha\right)\right) \eta_{2} \\
& +\frac{1}{2} I_{v}\left(\frac{1}{l}\left(\frac{\eta_{1}}{\cos \alpha}-\eta_{2}+\eta_{3} \tan \alpha\right)\right)+\frac{1}{2} m_{s}\left(\left(\eta_{1} \tan \alpha+\frac{\eta_{3}}{\cos \alpha}\right)^{2}+\eta_{1}^{2}\right) \\
& +\frac{1}{2} I_{s}\left(\frac{1}{l}\left(\frac{\eta_{1}}{\cos \alpha}-\eta_{2}+\eta_{3} \tan \alpha\right)+\eta_{4}\right)^{2}
\end{aligned}
$$

where $m_{v}$ is the mass of the vehicle without the front virtual wheel and the steering mechanism which are denoted by $m_{s}$, $I_{v}$ and $I_{s}$ are moment of inertia of the vehicle body and the front virtual steering wheel about a vertical axes, and $\mathbf{d}=\mathbf{P G}$ is the distance vector between point $P$ and the vehicle center of mass $G$.

Finally, using (12), (14), (15), (16), (17), (19) and (25), the dynamic equations of motion of the vehicle are obtained in the following matrix form

$$
M(q) \dot{\eta}_{b}+h\left(q, \eta_{b}\right)=\Gamma
$$

where

$$
M=\left[\begin{array}{cc}
m_{v}+\frac{m_{s} l^{2}+\left(I_{v}+I_{s}\right) \sin ^{2} \alpha}{l^{2} \cos ^{2} \alpha} & \frac{I_{s} \tan \alpha}{l} \\
\frac{I_{s} \tan \alpha}{l} & I_{s}
\end{array}\right]
$$

is $2 \times 2$ symmetric matrix,

$$
h=\left[\begin{array}{c}
\frac{\left(I_{v}+I_{s}+m_{s} l^{2}\right) \eta_{3} \eta_{4} \tan \alpha}{l^{2} \cos ^{2} \alpha} \\
\frac{I_{s} \eta_{3} \eta_{4}}{l \cos ^{2} \alpha}
\end{array}\right],
$$

and $\Gamma=\left[\Gamma_{3}, \Gamma_{4}\right]^{T}$ is a $2 \times 1$ vector of the generalized forces associated with the quasi-coordinates $\pi_{S},(s=3,4)$.

The generalized forces $\Gamma=\left[\Gamma_{3}, \Gamma_{4}\right]^{\mathrm{T}}$ associated with the quasi-coordinates $\pi_{s}(s=3,4)$ are related to the generalized forces $Q=\left[Q_{1}, Q_{2}, Q_{3}, Q_{4}\right]^{T}$ associated with the generalized coordinates $q$ according to the following relationships

$$
\Gamma=C^{T} Q \text {. }
$$

Finally, the mathematical model of the vehicle is obtained as composition of (7) and (26) in the form

$$
\begin{aligned}
& \dot{q}=C(q) \eta_{b} \\
& M(q) \dot{\eta}_{b}+h\left(q, \eta_{b}\right)=C^{T} Q
\end{aligned}
$$




\section{PROBLEM FORMULATION}

In this paper, we consider autonomous lane change maneuver without any information obtained from road infrastructure or inter-vehicle communication. The steering commands for the controlled vehicle are set according to the relative position and orientation with respect to the stopped (overtaken) car and, in that way, the vehicle accomplishes a lane change (obstacle avoidance-type) maneuver with respect to the stopped vehicle instead of the road. Assume that the automated vehicle has to pass the preceding car, which is at distance $e_{x}^{f}$ ahead, (Fig. 3).

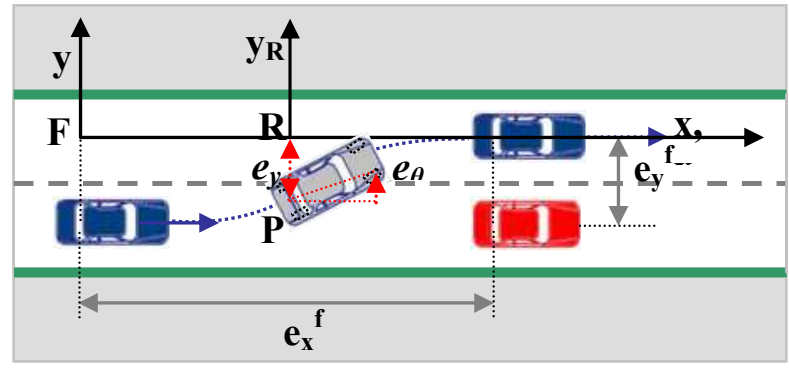

Figure 3. Schematic plan view of a lane change maneuver

Starting with some initial conditions, the automated vehicle diverts from its lane and tracks a given desired trajectory for a given time period and has to reach a preselected position on the left side (at distance $e_{y}^{f}$ ) of the stopped vehicle, (Fig. 3). The maneuver may be also considered as a first phase of an overtaking maneuver.

\section{A. Error Kinematics}

The path tracking geometry used in this paper is represented in Fig. 3. Consider a front-wheel steering vehicle moving on a flat surface. A moving reference coordinate frame $R x_{R} y_{R}$ is defined such that the $x_{R}$ axis coincides with xaxis of the inertial frame Fxyz. The $x_{R}$ axis is oriented in the direction of motion, which is parallel to the orientation of the stopped vehicle, but shifted at distance $e_{y}^{f}$. The $y_{R}$ axis of the moving reference frame passes through the reference point $P$ of the vehicle. The position and orientation of the robot vehicle with respect to the $R x_{R} y_{R}$ are denoted by $e_{y}$ and $e_{\theta}$, respectively. We suppose that the vehicle velocity $v_{P x}(t)$ is time-varying, bounded, but is always greater than zero, i.e., $0<v_{P x} \leq v_{P x}^{\max }$. Based on the assumption of pure rolling without lateral sliding of the wheels, the kinematic model for the lateral motion of the vehicle can be written in the form [12]

$$
\begin{aligned}
& \dot{e}_{y}=v_{P x}(t) \sin e_{\theta} \\
& \dot{e}_{\theta}=\frac{v_{P x}(t)}{l} \tan \alpha . \\
& \dot{\alpha}=\omega_{\alpha}
\end{aligned}
$$

\section{B. Trajectory Planning}

In this paper, we assign the desired trajectory in the form of cycloid which we define in parametric form in the time interval $t \in\left[0, t_{f}\right]$ as follows

$$
e_{x}^{d}(t)=\frac{e_{x}^{f}}{t_{f}} t ; \quad e_{y}^{d}(t)=e_{y}^{f}\left\{1-\left[\frac{t}{t_{f}}-\frac{1}{2 \pi} \sin \left(\frac{2 \pi}{t_{f}} t\right)\right]\right\} .
$$

The constants $e_{x}^{f}$ and $e_{y}^{f}$ in (32), (Fig. 3), are determined from considerations of safety driving, sensor requirements, and vehicle steering and acceleration capabilities. While the displacement $e_{y}^{f}$ at lateral direction is determined by considerations of safe driving and available free place on the left side of the stopped vehicle, the displacement $e_{x}^{f}$ at longitudinal direction is determined by lateral acceleration requirements and vehicle steering capabilities (maximum steering angle). At low speed operation of the automated vehicles in urban environments, parks and district areas, the steering capabilities of the vehicle become an important consideration in determining the displacement $e_{x}^{f}$ at longitudinal direction. The minimal value of $e_{x}^{f}$ could be roughly determined by considering a circular desired trajectory with maximum steering angle $\left|\alpha_{\max }\right|$. In this case, it is easy to determine that $e_{x \min }^{f}=e_{y}^{f} \sqrt{3}$, where $e_{y}^{f}=l / \tan \left|\alpha_{\max }\right|$. Given a cycloidal desired trajectory defined as in (32), the time $t_{f}$ needed for performing the maneuver is determined from the relationship: $t_{f}=e_{x}^{f} / v_{P x}(0)$, where $v_{P x}(0)$ is the vehicle velocity at $t=0$. It should be pointed out that at $t=0$ and $t=t_{f}$ the vehicle velocity has the same value, i.e., $v_{P x}(0)=v_{P x}\left(t_{f}\right)$, but during the maneuver, the velocity is not constant. A specific feature of the cycloidal trajectory is that there is not discontinuous acceleration, and therefore, infinite instantaneous jerk at the boundary points of the time interval.

\section{Problem Statement}

In order to design the vehicle steering controller, we assume that we are able to measure the error coordinates $e_{y}$ and $e_{\theta}$, the front-wheel steering angle $\alpha$ and angular velocity $\omega_{\alpha}$, as well the vehicle longitudinal speed $v_{P x}$. The vehicle acceleration is not available for feedback control. Given the inter-vehicle kinematics in error coordinates (31), the vehicle dynamic model (30), and the desired trajectory (32), the control objective is to track the trajectory (32) with the reference point $P$ of the vehicle.

\section{AdAPTIVE SteEring CONTROLleR}

The steering control scheme proposed in this paper, has a hierarchical two-layer structure based on the principle of decomposition of the control problem: an upper level kinematics-based path tracking control loop and a lower level steering dynamics-based control loop. The upper level controller provides a reference signal to the lower level controller which have to be tracked.

\section{A. Kinematics-Based Controller}

In order to design the upper (kinematics-based) controller, we make the following change of coordinates

$$
y_{e}=e_{y}-e_{y}^{d}
$$

where the error coordinate $e_{y}$ is the lateral error of the vehicle with respect to the moving frame $R x_{R} y_{R}$ and $e_{y}{ }^{d}$ is 
defined by (32). The model (31) for the error kinematics is redefined in terms of new coordinate (33) as follows

$$
\begin{aligned}
& \dot{y}_{e}=v_{P x}(t) \sin e_{\theta}-\dot{e}_{y}^{d} \\
& \dot{e}_{\theta}=\frac{v_{P x}(t)}{l} \tan \alpha \\
& \dot{\alpha}=\omega_{\alpha}
\end{aligned} .
$$

Assuming that the time-varying vehicle velocity $v_{P x}(t)$ is bounded (as well its derivatives) and does not converge to zero, we propose an inverse kinematics-type control law in the form

$$
\begin{aligned}
\omega_{\alpha} & =\frac{l \cos ^{2} \alpha}{v_{P x}^{2} \cos e_{\theta}}\left(\frac{v_{P x}^{3} \sin e_{\theta} \tan ^{2} \alpha}{l^{2}}-k_{2} \frac{v_{P x}^{2} \tan \alpha \cos e_{\theta}}{l}\right. \\
& \left.-k_{1} v_{P x} \sin e_{\theta}-k_{0} y_{e}+\dddot{e}_{y}^{d}+k_{2} \ddot{e}_{y}^{d}+k_{1} \dot{e}_{y}^{d}\right)
\end{aligned}
$$

where $k_{0}, k_{1}$ and $k_{2}$ are positive constants. The control law (35) is valid for $\left|e_{\theta}\right| \neq \pi / 2$ which is always satisfied from practical point of view during a lane change maneuver. We note that the control law (35) does not contain derivatives of the vehicle velocity $v_{P x}(t)$. Applying the control (35) to the system (34), the closed-loop system can be written in the form $\left(y_{1}:=y_{e}\right)$

$$
\dot{y}=A y+\xi
$$

where $y=\left[y_{1}, y_{2}, y_{3}\right]^{T}$. The matrix $A$ is a Hurwitz matrix and has the form

$$
A=\left[\begin{array}{ccc}
0 & 1 & 0 \\
0 & 0 & 1 \\
-k_{0} & -k_{1} & -k_{2}
\end{array}\right] .
$$

The system (36) can be viewed as a perturbation of a stable linear time-invariant system where the perturbation $\xi=h\left(y, v_{P x}, \dot{v}_{P x}\right)+\Delta_{e y}^{d}\left(\dot{e}_{y}^{d}, \ddot{e}_{y}^{d}, v_{P_{x}}, \dot{v}_{P_{x}}\right)$ is composed of two terms. The first term, $h$, is a vanishing perturbation $(h(0, t)=$ $0)$ ), and satisfies a linear growth bound $\|h\| \leq \zeta\|y\|$. The second term $\Delta_{e y}^{d}$, does not depend on the state variables $y$, and is a uniformly bounded function of time that satisfies $\left\|\Delta_{e y}^{d}\right\| \leq \delta$. In this case, the two perturbations are treated differently. Detailed stability analysis involves precise determination of the bounds $\zeta$ and $\delta$ for the perturbation terms and is left for future work. However, preliminary results based on the worst-case analysis show that with some constraints imposed on $\dot{v}_{P x}$, ultimate boundness of the overall system (36) can be claimed.

\section{B. Dynamics-Based Controller}

In this paper, we design an adaptive controller for the lower level steering dynamics control loop by using the method of Slotine [13]. For this end, we use the second equation from (26) of the vehicle dynamic model developed in Section 2, but we neglect the term associate with $\dot{v}_{P x}$. However, we retain the term which includes the time-varying vehicle velocity. Introducing the active torques associated with the generalized coordinates, the second equation of (26) is rewritten as

$$
\dot{\omega}_{\alpha}=-\sigma_{v} \omega_{\alpha}+\sigma_{p} \omega_{\alpha}+\psi \tau
$$

where $\sigma_{v}=\frac{v_{P x}}{l \cos ^{2} \alpha} ; \quad \psi=\frac{1}{I_{s}} ; \quad \sigma_{p}=k_{f} \psi ;$

$\tau$ is the driving torque, and $k_{f}$ is a coefficient of friction. The moment of inertia $I_{s}$ of the front steering wheel and the friction coefficient $k_{f}$ are unknown constant parameters. The desired performance of the control system for the steering dynamics is chosen to be as follows

$$
\dot{\omega}_{\alpha}^{d}=-c_{d}\left(\omega_{\alpha}^{d}-\omega_{\alpha}^{r}\right)
$$

where $c_{d}$ is a positive constant and $\omega_{\alpha}^{r}$ is the reference angular velocity obtained from the upper level control loop (eq. (35)). We introduce the following constants which include the unknown parameters

$$
\lambda_{r}=\frac{c_{d}}{\psi} ; \quad \lambda_{m}=\frac{\sigma_{p}-c_{d}}{\psi} .
$$

The parameter errors are denoted by

$$
\tilde{\lambda}_{r}=\hat{\lambda}_{r}-\lambda_{r} ; \quad \tilde{\lambda}_{m}=\hat{\lambda}_{m}-\lambda_{m}
$$

where $\hat{\lambda}_{r}$ and $\hat{\lambda}_{m}$ are the parameter estimates. The tracking error is defined by

$$
e_{\omega \alpha}=\omega_{\alpha}-\omega_{\alpha}^{d} .
$$

We choose a control law for (38) given by

$$
\tau=\hat{\lambda}_{r}\left(\omega_{r}^{\alpha}+\frac{\sigma_{v}}{c_{d}}\right)+\hat{\lambda}_{m} \omega_{\alpha} .
$$

Consider a positive definite, radially unbounded function $V$ of the form

$$
V=\frac{1}{2} e_{\omega \alpha}^{2}+\frac{\psi}{2 \mu_{y}} \widetilde{\lambda}_{m}^{2}+\frac{\psi}{2 \mu_{r}} \widetilde{\lambda}_{r}^{2}
$$

where $\mu_{y}$ and $\mu_{r}$ are positive gains. The derivative of $V$ along the closed-loop system trajectories is given by

$$
\dot{V}=c_{d} e_{\omega \alpha}^{2}+\psi \tilde{\lambda}_{m}\left(e_{\omega \alpha}+\frac{1}{\mu_{m}} \dot{\hat{\lambda}}_{m}\right)+\psi \tilde{\lambda}_{r}\left(e_{\omega \alpha}\left(\omega_{\alpha}^{r}+\frac{\sigma_{v}}{c_{d}} \omega_{\alpha}\right)+\frac{1}{\mu_{r}} \dot{\hat{\lambda}}_{r}\right) .
$$

We design an adaptive update law as follows

$$
\begin{aligned}
& \dot{\hat{\lambda}}_{m}=-\mu_{m} e_{\omega \alpha} \omega_{\alpha} \\
& \dot{\hat{\lambda}}=-\mu_{r} e_{\omega \alpha}\left(\omega_{\alpha}^{r}+\frac{\sigma_{v}}{c_{d}} \omega_{\alpha}\right) .
\end{aligned}
$$

Substituting (46) into (45), $\dot{V}$ results in

$$
\dot{V}=-c_{d} e_{\omega \alpha}^{2} \leq 0 \text {. }
$$

The closed-loop error dynamics is obtained in the form

$$
\dot{e}_{\omega \alpha}=-c_{d} e_{\omega \alpha}+\psi \tilde{\lambda}_{m} \omega_{\alpha}+\psi \tilde{\lambda}_{r}\left(\omega_{\alpha}^{r}+\frac{\sigma_{v}}{c_{d}} \omega_{\alpha}\right) .
$$

From (44) and (47), it follows that the error dynamics is stable, and $e_{\omega \alpha}, \tilde{\lambda}_{r}$ and $\tilde{\lambda}_{m}$ are bounded. Furthermore, from 
the boundness of $e_{\omega \alpha}, \tilde{\lambda}_{r}$ and $\tilde{\lambda}_{m}$, it follows the boundness of $\dot{e}_{\omega \alpha}$ (eq. (48)). By using the Barbalat's lemma [13], it follows that the tracking error $e_{\omega \alpha}$ is asymptotically stable, since $\dot{V}$ is uniformly continuous ( $\ddot{V}=-2 e_{\omega \alpha} \dot{e}_{\omega \alpha}$ is bounded).

\section{Simulation Results}

Simulation results are presented by using MATLAB to illustrate the performance of the proposed two-layer steering controller. The longitudinal vehicle base of the automated vehicle was chosen to be $l=1.5 \mathrm{~m}$. The velocity $v_{P x}(0)$ in the beginning of the maneuver was $1.5 \mathrm{~m} / \mathrm{s}$. The vehicle has to arrive in the end of the maneuver at the same velocity. The constants $e_{x}^{f}$ and $e_{y}^{f}$ of the desired cycloidal trajectory were $2.5 \mathrm{~m}$ and $7 \mathrm{~m}$, respectively. The initial values for the estimates of constant parameters $\hat{I}_{s}(0)$ and $\hat{k}_{f}(0)$ for the adaptive update control law were chosen to be equal to zero, indicating no a priory knowledge. In Fig. 4 and Fig. 5 are shown simulation results for the planar path, drown by the automated vehicle during the lane change maneuver and evolution in time of the vehicle velocity, respectively.

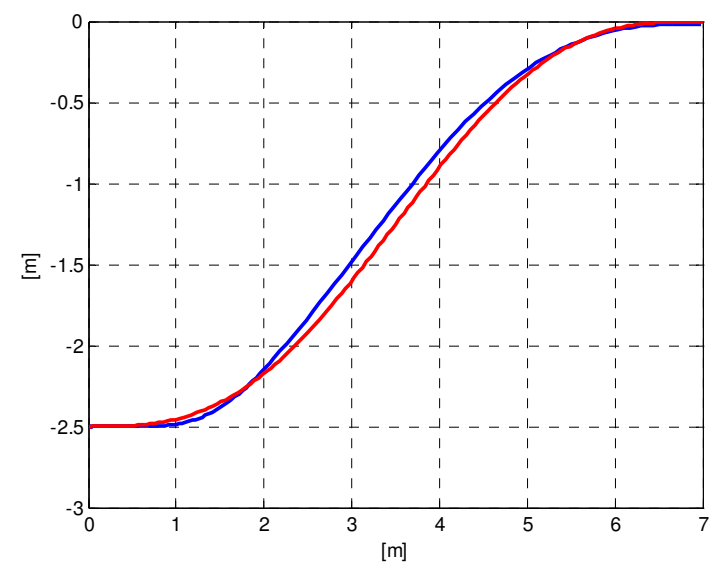

Figure 4. Planar path drown by the reference point $\mathrm{P}$ of the automated vehicle (blue line) and desired cycloidal path (red line)

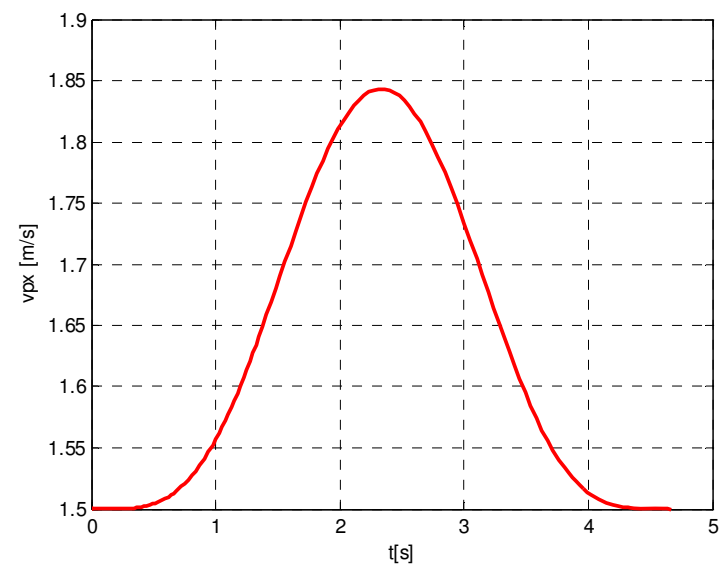

Figure 5. Evolution in time of the vehicle velocity $v_{P x}$, during the lane change maneuver
The maximal value of the tracking error $y_{e}^{\max }$ is obtained to be equal to $0.081 \mathrm{~m}$. The results of the simulation confirm the validity of the proposed controller.

\section{CONCLUSION}

In this paper, a two-layer nonlinear adaptive steering controller for an autonomous lane change maneuver with respect to a stopped vehicle has been presented. We consider the problem of autonomous lane change without the use of road infrastructure with only the current inter-vehicle position and orientation available for feedback control obtained from onboard sensors. A dynamic model of the vehicle using the Boltzmann-Hamel method in quasicoordinates for nonholonomic systems was derived. The lane change maneuver was investigated as a tracking problem with respect to desired cycloidal trajectory, which is generated in real time. An update control law was designed that allows tracking the desired trajectories in the presence of unknown inertial parameters of the vehicle. Simulation results illustrate the performance of the proposed controller. Our future work will focus on the implementation of the proposed controller on the experimental vehicle (Fig. 1) for personal transport developed at INRIA.

\section{REFERENCES}

[1] R. Rajamani, Vehicle dynamics and control, Springer, 2012.

[2] J. Balla, "Dynamics of mounted automatic cannon on track vehicle," Int. J. Math. Models and Methods in Appl. Sciences," Issue 3, Volume 5, pp. 423-432, 2011.

[3] P. Petrov and M. Parent, "Dynamic modeling and adaptive motion control of a two-wheeled self-balancing vehicle for personal transport," in Proc. $13^{\text {th }}$ Int. IEEE Annual Conf. Intell. Transp. Systems, Madeira, Portugal, 2010 pp.1113-115.

[4] W. Chee and M. Tomizuka, "Vehicle lane change maneuver in automated highway systems," California PATH Research Report, UCB-ITS-PRR-94-92, 1994.

[5] O. Mokhiamar and M. Abe, "Active wheel steering and yaw moment control combination to maximize stability as well as vehicle responsiveness during quick lane change for active vehicle handling safety," in Proc. Instn. Mech. Engrs, vol 216, Part D: J. Automobile Engineering, 2002, pp. 115-124.

[6] Resende and F. Nashashibi, "Real-time dynamic trajectory planning for highly automated driving in highways," in IEEE Conf. Intel. Transp. Systems, 2010, pp. 653-658.

[7] C. Laugier, "Towards autonomous vehicles for future intelligent transportation systems," available at: http:// citeseerx.ist.psu.edu/viewdoc/summary?doi=10.1.1.52.4080.

[8] C. Hatipoglu, K. Redmill, and U. Ozguner, "Steering and lane change: A working system," in Proc. Intel. Trans. Systems, Boston, USA, 1997, pp. 272-277.

[9] C. Hatipoglu, Ü. Özgüner, and K. Redmill, "Automated Lane Change Controller Design,” IEEE Trans. Intel. Trans. Systems, Vol. 4, No. 1, pp. 13-22, 2003.

[10] J. Neimark and N. Fufaev, Dynamics of nonholonomic systems, Trans. Mathematical Monograph, Vol. 33, Math. Society, 1972.

[11] E. Jarzebowska, "Quasi-coordinates based dynamics modeling and control design for nonholonomic systems," Nonlinear Analysis, 71, 2009, pp. 118-131.

[12] P.Petrov and M. Parent, "Nonlinear path control of a four-wheel steering vehicle," in Proc. $11^{\text {th }}$ Int Conf. Advanced Robotics, Coimbra, Portugal, 2003.

[13] J.-J. Slotine and W. Li, Applied Nonlinear Control, Prentice Hall, 1991. 